\title{
Physiological Maturity and Longevity of Pumpkin Seeds in Relation to Fruit Age and Duration of in situ Storage
}

\author{
Rajinder Singh ${ }^{1}$, Parvinder Singh ${ }^{2 *}$ and Tarsem Singh Dhillon ${ }^{3}$ \\ ${ }^{1}$ Director (Seeds), Punjab Agricultural University, Ludhiana, India \\ ${ }^{2}$ Krishi Vigyan Kendra, Amritsar, India \\ *Corresponding author
}



\section{A B S T R A C T}

An experiment was conducted in 2017 to study the seed quality of pumpkin (Cucurbita pepo L.) variety Punjab Samrat as aff ected by stage of fruit harvest and post harvest storage duration and to see their effect on longevity of the seed. Fruits were harvested 25, 35 and 45 days after anthesis (DAA) which were stored for $0,10,20$ and 30 days after each date of harvesting (DAH). These seeds were stored in cloth bags under ambient conditions for 9 months. The germination, speed of germination, seedling length, dry weight and vigour indices were better in fruits harvested at 45 DAA. Similarly seed quality was better when pre-storage duration were 30 days. The longevity of the seed also remained high in the mature seeds. The interaction effects revealed that germination percentage, speed of germination, seedling length, dry weight and vigour indices were better when fruits were harvested $45 \mathrm{DAA}$ and retained for at least 10 days in fruits before harvest. Therefore to get good quality seed and to retain its viability for longer period should be harvested 45 DAA and pre-stored for at least 10 days before its extraction.

\section{Introduction}

Pumpkin is an important cucurbit grown for its fleshy fruits in tropical and subtropical regions. Cucurbit seeds are consumed as snack in some parts of the world because their nutritive values are quite high. However, most of seed production is directed for propagation. However, seed production in this crop is comparatively complicated than in dry-seeded grain crops, because seed mature within the moist fruit and are often held at high moisture content for several weeks before harvest (Welbaum, 1999). The crop being monoecious exhibit male and female flowers and all the female flowers do not reach at physiological maturity simultaneously and even at the senescence stage of the plants, all the fruits do not reach the physiological maturity and as a result seed produced is 
mixture of mature and immature seed at the time of harvest (Nerson and Parris, 1988). This results in poor seed yield and low germination rate (Achigan et al., 2008).

In general, the three major phases during seed development are rapid gain in fresh weight, rapid dry weight accumulation and loss of fresh weight during drying. However, in cucurbits dry weight accumulation period is extended over longer period compared to dry seeded grain crop (Welbaum, 1999). Cucurbits seed maturity occurs over a large range of days after pollination and depends primarily on temperature during development and do not require desiccation in order to mature and transition from developmental to germinative mode (Nerson, 2007). The stage of maturity determines storage potential of seeds. Early harvested seeds are immature and poorly developed and are poor storer compared to seed harvested at proper physiological maturity (Singh and Lachanna, 1995).

Pumpkin fruits in mature stage can be kept for several days after harvest and it also act as natural store house for the seed. It is also argued that fruits that do not reach the physiological maturity but seeds may be kept in fruit for few days after detaching from the plant may allow the nutrients to transfer from pulp to seed results in production of good quality seed. Since the development of seed continues in the fleshy fruit due to continuous supply of nutrients from fruit to seeds, there is need to study various physiological and biochemical changes associated with various fruit age and pre-storage duration. As early harvested seeds may have poor storability and there is also need to study their storage potential. Thus keeping in view of the justification explained above, the present investigation was conducted to ascertain the effect of stage of fruit harvest and pre-storage duration for optimum seed viability and vigour in pumpkin seed over different period.

\section{Materials and Methods}

The seed crop of pumpkin variety Punjab Samrat was raised at the Seed Experimental Farm, O/o Director (Seeds), P.A.U. Ludhiana in 2017. Seed was sown in polythene bags in second week of February and transplanted in second week of March in 2017. Experiment was conducted in randomized block factorial design. The first factor was three harvest stages $(25,35$ and 45 days after pollination) and second factor was storage duration $(0,10$, 20 and 30 days after harvest). Each female flower was date-tagged at anthesis to monitor fruit age and fruit harvested at 25, 35 and 45 days after pollination (DAP) by insect and then stored for $0,10,20$ and 30 days at room temperature, before extracting the seeds. Two fruits per vine were retained. After that the seeds were extracted from these fruits, washed, dried under shade-net and stored. 5 fruits per replication in each treatment were selected for recording observation. The extracted seeds were kept in cloth bags under ambient conditions for 3, 6 and 9 months and data were recorded accordingly. Germination was recorded as per ISTA rules (Anonymous, 2011).Various physiological parameters viz; speed of germination (Raun et al., 2002), seedling length $(\mathrm{cm})$. Seedling dry weight $(\mathrm{g})$ and seedling vigour index Abdul baki and Anderson (1973) were recorded. Speed of germination was calculated as: $\Sigma(\mathrm{Gt} / \mathrm{Tt})$ (where $\mathrm{Gt}=$ number of seeds germinated or emerged on $\mathrm{t}^{\text {th }}$ day; $\mathrm{Tt}=$ number of days upto $\mathrm{t}^{\text {th }}$ day); Seedling vigour was calculated as: (percent germination $\mathrm{x}$ total seedling dry weight).

\section{Results and Discussion}

Data presented in Table 1 clearly indicated that stage of harvest had significant effect on germination of seed for freshly harvested seeds. Maximum germination $(85.17 \%)$ was observed when fruits were harvested 45 days 
after anthesis and germination did not decreased considerably when seeds were stored for 3, 6 and 9 months respectively. Germination at the end of 9 months of ambient storage was $81.75 \%$ when fruits were harvested 45 days after anthesis (DAA). However when fruits were harvested 25 and 35 days after anthesis resulted in poor storability. Similarly pre-storage duration significantly affected the germination. Highest germination (75.33\%) was observed when seeds were kept 30 days in fruit and lowest was observed when seeds were extracted immediately after fruit harvest. However when seeds were stored for 3, 6 and 9 months showed variation in germination. The seeds extracted after 30 days retained their germination $71.11 \%$ after 9 months of storage (Table 4) while seeds extracted immediately lost the germination considerably. The interaction effects clearly indicated that fruits harvested $45 \mathrm{DAA}$ and seed extracted 10,20 or 30 days had significantly no difference in germination and these seeds retained their longevity even 9 months after storage and were significantly better than other harvesting stages and prestorage durations. The fruits harvested at 25 DAA and seeds extracted immediately showed nil germination and their germination delayed gradually while fruits harvested 35 DAA and increased pre-storage duration significantly improved the germination. The increased germination as the harvesting delayed is directly related to physiological maturity of the seed. The filling of seed reserve material like starch, lipid, protein, sugars have been completely filled and the biochemical enzymes required for germination of the seed are better than physiological immature seed resulting in better germination. Similarly pre-storage duration improved the germination might be due to the transfer of assimilates from fruit pulp to seeds and as the duration increased the nutrients transmission also increased. Even the physiologically immature seeds showed increased germination after pre-storage duration. Huma et al., (2016) reported improved germination in pumpkin seed when harvested 45 DAA and kept in fruit for 30 days. Yao et al., (2012) reported similar findings in watermelon.

Speed of germination was also significantly better in case of fruits harvested 45 DAA while lowest speed of germination was recorded when fruit harvested 25 DAA. Storage conditions decreased the speed of germination considerably and lowest speed was recorded after 9 months of storage. However fruits harvested 45 DAA showed considerably better speed of germination. Prestorage durations no doubt improved the speed of germination and highest speed was recorded when seeds were extracted 30 days after storage and very poor speed of germination was observed when seeds were extracted immediately after harvest of fruits. Interaction effects revealed that fruits harvested 45 DAA and seed extracted 10, 20 or 30 days had significantly no difference in speed of germination and these seeds retained their longevity even 9 months after storage and were significantly better than other harvesting stages and pre-storage durations. The fruits harvested at 25 DAA and seeds extracted immediately showed nil germination and so speed of germination while fruits harvested $35 \mathrm{DAA}$ and increased pre-storage duration significantly improved the speed of germination. As the mature seeds were properly filled and very less unfilled seed are there may improve the speed of germination. Oztokat et al., (2004) proposed that in cucurbit crops (Citrullus lanatus) seed viability and germination percentage and seed vigour increased when the age of harvest was delayed from 28 to 49 days after anthesis. Tarus et al., 2017 also reported increased seed vigour in pumkin with delay in harvesting of the fruit. 
Table.1 Effect of stage of harvest and pre-storage duration on seed quality of pumpkin immediately after harvest

\begin{tabular}{|c|c|c|c|c|c|c|c|}
\hline $\begin{array}{l}\text { S. } \\
\text { No }\end{array}$ & Treatments & $\begin{array}{c}\text { Germination } \\
(\%)\end{array}$ & $\begin{array}{c}\text { Speed of } \\
\text { germination }\end{array}$ & $\begin{array}{c}\text { Seedling } \\
\text { length } \\
(\mathbf{c m})\end{array}$ & $\begin{array}{l}\text { Seedling } \\
\text { dry weight } \\
\text { (g) }\end{array}$ & $\begin{array}{l}\text { Vigour } \\
\text { index-I }\end{array}$ & $\begin{array}{c}\text { Vigour } \\
\text { index-II }\end{array}$ \\
\hline \multicolumn{8}{|c|}{ Stage of harvest } \\
\hline 1. & $\begin{array}{l}\mathrm{H}_{1}-25 \text { days } \\
\text { after anthesis }\end{array}$ & 21.67 & 1.34 & 9.82 & 0.44 & 307.30 & 13.26 \\
\hline 2. & $\begin{array}{c}\mathrm{H}_{2}-35 \text { days } \\
\text { after anthesis }\end{array}$ & 55.08 & 1.77 & 13.11 & 0.46 & 970.78 & 34.68 \\
\hline \multirow[t]{2}{*}{3.} & $\begin{array}{l}\mathrm{H}_{3}-45 \text { days } \\
\text { after anthesis }\end{array}$ & 85.17 & 2.89 & 18.76 & 0.81 & $\begin{array}{c}1614.8 \\
0\end{array}$ & 69.89 \\
\hline & C.D. $(0.05)$ & 3.12 & 0.11 & 0.39 & 0.02 & 60.44 & 2.52 \\
\hline \multicolumn{8}{|c|}{ Prestorage duration } \\
\hline 1. & $\begin{array}{c}\mathrm{S}_{0}- \\
\text { Immediate } \\
\text { extraction }\end{array}$ & 17.89 & 0.39 & 5.71 & 0.24 & 306.90 & 12.99 \\
\hline 2. & $\begin{array}{c}\mathrm{S}_{1}-\text { after } 10 \\
\text { days }\end{array}$ & 55.77 & 2.20 & 15.06 & 0.64 & 970.54 & 39.99 \\
\hline 3. & $\begin{array}{c}\mathrm{S}_{2}-\text { after } 20 \\
\text { days }\end{array}$ & 66.89 & 2.66 & 16.81 & 0.67 & 1193.95 & 47.52 \\
\hline \multirow[t]{2}{*}{4.} & $\begin{array}{c}\mathrm{S}_{3}-\text { after } 30 \\
\text { days }\end{array}$ & 75.33 & 2.74 & 18.00 & 0.73 & 1385.80 & 56.59 \\
\hline & C.D. $(0.05)$ & 3.60 & 0.13 & 0.45 & 0.023 & 69.79 & 2.91 \\
\hline \multicolumn{8}{|c|}{ Interactions } \\
\hline 1. & $\mathrm{H}_{1} \times \mathrm{S}_{0}$ & 0.00 & 0.00 & 0.00 & 0.00 & 0.00 & 0.00 \\
\hline 2. & $\mathrm{H}_{1} \times \mathrm{S}_{1}$ & 15.00 & 1.17 & 9.57 & 0.52 & 145.25 & 7.80 \\
\hline 3. & $\mathrm{H}_{1} \times \mathrm{S}_{2}$ & 30.00 & 20.3 & 13.32 & 0.58 & 398.56 & 17.42 \\
\hline 4. & $\mathrm{H}_{1} \times \mathrm{S}_{3}$ & 41.67 & 2.15 & 16.38 & 0.66 & 685.41 & 27.82 \\
\hline 5. & $\mathrm{H}_{2} \times \mathrm{S}_{0}$ & 0.00 & 0.00 & 0.00 & 0.00 & 0.00 & 0.00 \\
\hline 6. & $\mathrm{H}_{2} \times \mathrm{S}_{1}$ & 57.00 & 2.13 & 16.51 & 0.56 & 946.00 & 32.08 \\
\hline 7. & $\mathrm{H}_{2} \times \mathrm{S}_{2}$ & 75.00 & 2.042 & 17.77 & 0.60 & 1333.83 & 45.01 \\
\hline 8. & $\mathrm{H}_{2} \times \mathrm{S}_{3}$ & 88.33 & 2.53 & 18.15 & 0.70 & 1603.30 & 61.64 \\
\hline 9. & $\mathrm{H}_{3} \times \mathrm{S}_{0}$ & 53.67 & 1.16 & 17.13 & 0.73 & 920.70 & 38.98 \\
\hline 10. & $\mathrm{H}_{3} \times \mathrm{S}_{1}$ & 95.33 & 3.32 & 19.10 & 0.84 & 1820.36 & 80.10 \\
\hline 11. & $\mathrm{H}_{3} \times \mathrm{S}_{2}$ & 95.67 & 3.52 & 19.33 & 0.84 & 1849.46 & 80.14 \\
\hline 12. & $\mathrm{H}_{3} \times \mathrm{S}_{3}$ & 96.00 & 3.56 & 19.47 & 0.84 & 1868.70 & 80.32 \\
\hline \multicolumn{2}{|c|}{ C.D. $(P=0.05)$} & 6.23 & 0.22 & 0.45 & 0.04 & 120.88 & 5.04 \\
\hline & C.V. & 6.82 & 6.58 & 3.35 & 4.19 & 7.40 & 7.57 \\
\hline
\end{tabular}


Table.2 Effect of stage of harvest and pre-storage duration on seed quality of pumpkin after 3 months of harvest

\begin{tabular}{|c|c|c|c|c|c|c|c|}
\hline $\begin{array}{l}\text { S. } \\
\text { No }\end{array}$ & Treatments & $\begin{array}{c}\text { Germination } \\
(\%)\end{array}$ & $\begin{array}{c}\text { Speed of } \\
\text { germination }\end{array}$ & $\begin{array}{l}\text { Seedling } \\
\text { length } \\
\text { (cm) }\end{array}$ & $\begin{array}{l}\text { Seedling } \\
\text { dry } \\
\text { weight } \\
\text { (g) }\end{array}$ & $\begin{array}{l}\text { Vigour } \\
\text { index-I }\end{array}$ & $\begin{array}{l}\text { Vigour } \\
\text { index-II }\end{array}$ \\
\hline \multicolumn{8}{|c|}{ Stage of harvest } \\
\hline 1. & $\begin{array}{l}\mathrm{H}_{1}-25 \text { days } \\
\text { after anthesis }\end{array}$ & 20.92 & 1.32 & 9.75 & 0.43 & 295.82 & 12.65 \\
\hline 2. & $\begin{array}{l}\mathrm{H}_{2}-35 \text { days } \\
\text { after anthesis }\end{array}$ & 54.50 & 1.75 & 13.03 & 0.46 & 954.93 & 33.98 \\
\hline \multirow[t]{2}{*}{3.} & $\begin{array}{l}\mathrm{H}_{3}-45 \text { days } \\
\text { atter anthesis }\end{array}$ & 84.42 & 2.87 & 18.76 & 0.81 & 1602.03 & 69.07 \\
\hline & C.D. (0.05) & 3.19 & 0.11 & 0.39 & 0.018 & 56.41 & 2.28 \\
\hline \multicolumn{8}{|c|}{ Pre-storage duration } \\
\hline 1. & $\begin{array}{c}\mathrm{S}_{0}- \\
\text { Immediate } \\
\text { extraction }\end{array}$ & 17.22 & 0.38 & 5.69 & 0.24 & 294.44 & 12.41 \\
\hline 2. & $\begin{array}{c}\mathrm{S}_{1}-\text { after } 10 \\
\text { days }\end{array}$ & 55.33 & 2.18 & 15.02 & 0.64 & 962.67 & 39.60 \\
\hline 3. & $\begin{array}{c}\mathrm{S}_{2}-\text { after } 20 \\
\text { days }\end{array}$ & 66.11 & 2.64 & 16.72 & 0.68 & 1179.35 & 46.72 \\
\hline \multirow[t]{2}{*}{4.} & $\begin{array}{c}\mathrm{S}_{3}-\text { after } 30 \\
\text { days }\end{array}$ & 74.44 & 2.73 & 17.96 & 0.73 & 1367.25 & 55.54 \\
\hline & C.D. (0.05) & 3.69 & 0.13 & 0.45 & 0.021 & 65.13 & 2.64 \\
\hline \multicolumn{8}{|c|}{ Interactions } \\
\hline 1. & $\mathrm{H}_{1} \times \mathrm{S}_{0}$ & 0.00 & 0.00 & 0.00 & 0.00 & 0.00 & 0.00 \\
\hline 2. & $\mathrm{H}_{1} \times \mathrm{S}_{1}$ & 14.33 & 1.17 & 9.57 & 0.51 & 139.58 & 7.40 \\
\hline 3. & $\mathrm{H}_{1} \times \mathrm{S}_{2}$ & 28.33 & 2.00 & 13.13 & 0.57 & 371.79 & 16.32 \\
\hline 4. & $\mathrm{H}_{1} \times \mathrm{S}_{3}$ & 41.00 & 2.13 & 16.31 & 0.65 & 671.92 & 26.87 \\
\hline 5. & $\mathrm{H}_{2} \times \mathrm{S}_{0}$ & 0.00 & 0.00 & 0.00 & 0.00 & 0.00 & 0.00 \\
\hline 6. & $\mathrm{H}_{2} \times \mathrm{S}_{1}$ & 56.00 & 2.11 & 16.35 & 0.55 & 919.05 & 31.02 \\
\hline 7. & $\mathrm{H}_{2} \times \mathrm{S}_{2}$ & 74.67 & 2.39 & 17.63 & 0.59 & 1316.73 & 44.29 \\
\hline 8. & $\mathrm{H}_{2} \times \mathrm{S}_{3}$ & 87.33 & 2.50 & 18.13 & 0.69 & 1583.93 & 60.61 \\
\hline 9. & $\mathrm{H}_{3} \times \mathrm{S}_{0}$ & 51.67 & 1.14 & 17.07 & 0.72 & 883.33 & 37.22 \\
\hline 10. & $\mathrm{H}_{3} \times \mathrm{S}_{1}$ & 95.67 & 3.25 & 19.13 & 0.84 & 1829.37 & 80.38 \\
\hline 11. & $\mathrm{H}_{3} \times \mathrm{S}_{2}$ & 95.33 & 3.53 & 19.40 & 0.83 & 1849.53 & 79.54 \\
\hline \multirow[t]{3}{*}{12.} & $\mathrm{H}_{3} \times \mathrm{S}_{3}$ & 95.00 & 3.55 & 19.43 & 0.83 & 1845.90 & 79.16 \\
\hline & C.D. $(0.05)$ & 6.39 & 0.22 & 0.79 & 0.036 & 112.81 & 4.57 \\
\hline & C.V. & 7.08 & 6.51 & 3.36 & 3.71 & 7.01 & 7.00 \\
\hline
\end{tabular}


Table.3 Effect of stage of harvest and pre-storage duration on seed quality of pumpkin after 6 months of harvest

\begin{tabular}{|c|c|c|c|c|c|c|c|}
\hline $\begin{array}{l}\text { S. } \\
\text { No }\end{array}$ & Treatments & $\begin{array}{c}\text { Germination } \\
(\%)\end{array}$ & $\begin{array}{c}\text { Speed of } \\
\text { germination }\end{array}$ & $\begin{array}{l}\text { Seedling } \\
\text { length } \\
\text { (cm) }\end{array}$ & $\begin{array}{c}\text { Seedling } \\
\text { dry weight } \\
\text { (g) }\end{array}$ & $\begin{array}{l}\text { Vigour } \\
\text { index-I }\end{array}$ & $\begin{array}{c}\text { Vigour } \\
\text { index-II }\end{array}$ \\
\hline \multicolumn{8}{|c|}{ Stage of harvest } \\
\hline 1. & $\begin{array}{l}\mathrm{H}_{1}-25 \text { days } \\
\text { after anthesis }\end{array}$ & 19.67 & 1.32 & 9.67 & 0.43 & 276.30 & 11.84 \\
\hline 2. & $\begin{array}{l}\mathrm{H}_{2}-35 \text { days } \\
\text { after anthesis }\end{array}$ & 52.50 & 1.74 & 12.96 & 0.46 & 915.24 & 32.59 \\
\hline 3. & $\begin{array}{l}\mathrm{H}_{3}-45 \text { days } \\
\text { after anthesis }\end{array}$ & 83.58 & 2.85 & 18.68 & 0.81 & 1580.90 & 68.49 \\
\hline & C.D. $(0.05)$ & 2.66 & 0.085 & 0.37 & 0.017 & 48.69 & 2.00 \\
\hline \multicolumn{8}{|c|}{ Pre-storage duration } \\
\hline 1. & $\begin{array}{c}\mathrm{S}_{0}- \\
\text { Immediate } \\
\text { extraction }\end{array}$ & 16.56 & 0.37 & 5.64 & 0.24 & 281.11 & 11.88 \\
\hline 2. & $\begin{array}{c}\mathrm{S}_{1}-\text { after } 10 \\
\text { days }\end{array}$ & 53.67 & 2.16 & 14.91 & 0.63 & 931.63 & 38.42 \\
\hline 3. & $\begin{array}{c}\mathrm{S}_{2}-\text { after } 20 \\
\text { days }\end{array}$ & 64.44 & 2.63 & 16.62 & 0.67 & 1145.66 & 45.80 \\
\hline 4. & $\begin{array}{c}\mathrm{S}_{3}-\text { after } 30 \\
\text { days }\end{array}$ & 73.00 & 2.71 & 17.89 & 0.73 & 1338.19 & 54.47 \\
\hline & C.D. $(0.05)$ & 3.07 & 0.099 & 0.42 & 0.019 & 56.23 & 2.30 \\
\hline \multicolumn{8}{|c|}{ Interactions } \\
\hline 1. & $\mathrm{H}_{1} \times \mathrm{S}_{0}$ & 0.00 & 0.00 & 0.00 & 0.00 & 0.00 & 0.00 \\
\hline 2. & $\mathrm{H}_{1} \times \mathrm{S}_{1}$ & 13.33 & 1.16 & 9.40 & 0.51 & 126.80 & 6.84 \\
\hline 3. & $\mathrm{H}_{1} \times \mathrm{S}_{2}$ & 26.67 & 1.99 & 13.07 & 0.57 & 349.35 & 15.19 \\
\hline 4. & $\mathrm{H}_{1} \times \mathrm{S}_{3}$ & 38.67 & 2.11 & 16.20 & 0.65 & 629.07 & 25.33 \\
\hline 5. & $\mathrm{H}_{2} \times \mathrm{S}_{0}$ & 0.00 & 0.00 & 0.00 & 0.00 & 0.00 & 0.00 \\
\hline 6. & $\mathrm{H}_{2} \times \mathrm{S}_{1}$ & 52.67 & 2.10 & 16.30 & 0.55 & 860.93 & 28.96 \\
\hline 7. & $\mathrm{H}_{2} \times \mathrm{S}_{2}$ & 72.00 & 2.38 & 17.50 & 0.60 & 1260.67 & 42.91 \\
\hline 8. & $\mathrm{H}_{2} \times \mathrm{S}_{3}$ & 85.33 & 2.49 & 18.03 & 0.69 & 1539.37 & 58.53 \\
\hline 9. & $\mathrm{H}_{3} \times \mathrm{S}_{0}$ & 49.67 & 1.12 & 16.93 & 0.72 & 843.33 & 35.64 \\
\hline 10. & $\mathrm{H}_{3} \times \mathrm{S}_{1}$ & 95.00 & 3.22 & 19.03 & 0.84 & 1807.17 & 79.49 \\
\hline 11. & $\mathrm{H}_{3} \times \mathrm{S}_{2}$ & 94.67 & 3.52 & 19.30 & 0.84 & 1826.97 & 79.30 \\
\hline \multirow[t]{3}{*}{12.} & $\mathrm{H}_{3} \times \mathrm{S}_{3}$ & 95.00 & 3.53 & 19.43 & 0.84 & 1846.13 & 79.54 \\
\hline & C.D. (0.05) & 5.32 & 0.17 & 0.73 & 0.034 & 97.38 & 3.99 \\
\hline & C.V. & & 5.13 & 3.14 & 3.51 & 6.22 & 6.26 \\
\hline
\end{tabular}


Table.4 Effect of stage of harvest and pre-storage duration on seed quality of pumpkin after 9 months of harvest

\begin{tabular}{|c|c|c|c|c|c|c|c|}
\hline $\begin{array}{l}\text { S. } \\
\text { No }\end{array}$ & Treatments & $\begin{array}{c}\text { Germination } \\
(\%)\end{array}$ & $\begin{array}{c}\text { Speed of } \\
\text { germination }\end{array}$ & $\begin{array}{c}\text { Seedling } \\
\text { length } \\
\text { (cm) }\end{array}$ & $\begin{array}{c}\text { Seedling } \\
\text { dry } \\
\text { weight (g) }\end{array}$ & $\begin{array}{l}\text { Vigour } \\
\text { index-I }\end{array}$ & $\begin{array}{l}\text { Vigour } \\
\text { index-II }\end{array}$ \\
\hline \multicolumn{8}{|c|}{ Stage of harvest } \\
\hline 1. & $\begin{array}{l}\mathrm{H}_{1}-25 \text { days } \\
\text { after anthesis }\end{array}$ & 18.25 & 1.29 & 9.53 & 0.43 & 252.99 & 10.81 \\
\hline 2. & $\begin{array}{l}\mathrm{H}_{2}-35 \text { days } \\
\text { after anthesis }\end{array}$ & 51.25 & 1.73 & 12.83 & 0.45 & 885.45 & 31.53 \\
\hline \multirow[t]{2}{*}{3.} & $\begin{array}{l}\mathrm{H}_{3}-45 \text { days } \\
\text { after anthesis }\end{array}$ & 81.75 & 2.83 & 18.55 & 0.80 & 1536.28 & 66.45 \\
\hline & C.D. (0.05) & 2.84 & 0.086 & 0.37 & 0.017 & 55.08 & 2.18 \\
\hline \multicolumn{8}{|c|}{ Pre-storage duration } \\
\hline 1. & $\begin{array}{c}\mathrm{S}_{0}- \\
\text { Immediate } \\
\text { extraction }\end{array}$ & 15.67 & 0.37 & 5.62 & 0.24 & 264.87 & 11.10 \\
\hline 2. & $\begin{array}{c}\mathrm{S}_{1}-\text { after } 10 \\
\text { days }\end{array}$ & 52.00 & 2.13 & 14.68 & 0.63 & 891.24 & 37.03 \\
\hline 3. & $\begin{array}{c}\mathrm{S}_{2}-\text { after } 20 \\
\text { days }\end{array}$ & 62.89 & 2.61 & 16.58 & 0.66 & 1119.67 & 44.19 \\
\hline \multirow[t]{2}{*}{4.} & $\begin{array}{c}\mathrm{S}_{3}-\text { after } 30 \\
\text { days }\end{array}$ & 71.11 & 2.69 & 17.66 & 0.72 & 1290.52 & 52.73 \\
\hline & C.D. (0.05) & 3.28 & 0.099 & 0.43 & 0.019 & 63.60 & 2.51 \\
\hline \multicolumn{8}{|c|}{ Interactions } \\
\hline 1. & $\mathrm{H}_{1} \times \mathrm{S}_{0}$ & 0.00 & 0.00 & 0.00 & 0.00 & 0.00 & 0.00 \\
\hline 2. & $\mathrm{H}_{1} \times \mathrm{S}_{1}$ & 12.33 & 1.13 & 9.27 & 0.51 & 115.43 & 6.27 \\
\hline 3. & $\mathrm{H}_{1} \times \mathrm{S}_{2}$ & 24.67 & 1.96 & 12.90 & 0.56 & 319.60 & 13.80 \\
\hline 4. & $\mathrm{H}_{1} \times \mathrm{S}_{3}$ & 36.00 & 2.07 & 15.93 & 0.64 & 576.93 & 23.16 \\
\hline 5. & $\mathrm{H}_{2} \times \mathrm{S}_{0}$ & 0.00 & 0.00 & 0.00 & 0.00 & 0.00 & 0.00 \\
\hline 6. & $\mathrm{H}_{2} \times \mathrm{S}_{1}$ & 51.00 & 2.08 & 16.00 & 0.54 & 820.00 & 27.73 \\
\hline 7. & $\mathrm{H}_{2} \times \mathrm{S}_{2}$ & 70.67 & 2.37 & 17.63 & 0.59 & 1247.53 & 41.44 \\
\hline 8. & $\mathrm{H}_{2} \times \mathrm{S}_{3}$ & 83.33 & 2.47 & 17.68 & 0.68 & 1474.27 & 56.94 \\
\hline 9. & $\mathrm{H}_{3} \times \mathrm{S}_{0}$ & 47.00 & 1.10 & 16.87 & 0.71 & 794.60 & 33.29 \\
\hline 10. & $\mathrm{H}_{3} \times \mathrm{S}_{1}$ & 92.67 & 3.19 & 18.77 & 0.83 & 1738.30 & 77.09 \\
\hline 11. & $\mathrm{H}_{3} \times \mathrm{S}_{2}$ & 93.33 & 3.51 & 19.20 & 0.83 & 1791.87 & 77.33 \\
\hline \multirow[t]{3}{*}{12.} & $\mathrm{H}_{3} \times \mathrm{S}_{3}$ & 94.00 & 3.53 & 19.37 & 0.83 & 1820.37 & 78.07 \\
\hline & C.D. $(0.05)$ & 5.68 & 0.17 & 0.75 & 0.034 & 110.16 & 4.35 \\
\hline & C.V. & 6.66 & 5.21 & 3.24 & 3.57 & 7.30 & 7.09 \\
\hline
\end{tabular}


Huma et al., 2016 also reported increased speed of germination of pumpkin seed with delayed harvest and increased pre-storage duration. Similar results were reported by Yao et al., 2012.

Seedling length and seedling dry weight were also significantly better when fruits harvested 45 DAA and minimum was observed when harvested 25 DAA. As the storage period increased the seedling length and dry weight decreased considerably (Table 2, 3, 4) indicating that less mature seeds loose the vigour fast than mature seeds. Pre-storage duration also affected the seedling length and dry weight significantly. The seeds extracted 30 days after storage had maximum seedling length and dry weight and it was significantly better than other storage durations. The minimum seedling length and dry weight was observed when seeds were extracted immediately after fruit harvest. Longevity of the seed also reduced with storage duration. Interaction effects clearly revealed that fruits harvested at 45 DAA and seed extracted after 10,20 or 30 days exhibited maximum seedling length and dry weight. However fruits harvested 35 DAA and seed extracted after 20 or 30 days exhibited significant seed ling length and dry weight. Seedling vigour indices indicate the capacity of the seedling to develop into main plant when sown under appropriate conditions. Vigour was also better with fruits harvested at 45 DAA and prestorage duration revealed that 30 days period had significantly better vigour indices than other storage durations. Storage durations also reduced the vigour considerably but physiologically mature seeds tends to retain vigour more than the immature seeds. The interaction effects revealed highest vigour of the seeds when fruits harvested 45 DAA and retained for at least 10 days before extraction from the fruit. Seedling length, dry weight and vigour indices are totally dependent upon the seed reserve material. As the harvesting time of the fruit delayed the vigour of seed decreased that clearly indicate that seed had reached the physiological maturity stage and had gained the maximum dry weight. Similarly pre-storage duration improved the seed vigour might be the transfer of nutrients from fruit pulp to immature seeds. The dry or immature fruits may act as store house for the seed and seed can be kept for longer period in the fruit without any loss to seed viability but may transfer the nutrients to the seed. Similar results were reported by Wilson and Mcdonald (1986) and Karklelienen et al., 2008.

The present study concluded that to get good quality seed of pumpkin and to retain its viability for longer period, it should be harvested 45 days after anthesis and should be kept at least 10 days in fruit before extraction.

\section{References}

Abdul-Baki A S and Anderson J D (1973). Vigour determination in soyabean by multiple criteria. Crop Sci13:630-33

Achigan-Dako EG, Fagbemissi F, Avohou HT, Vodouhe RS AND Ahanchede OCA(2008). Importance and practices of Egusi crops (Citrullus lanatus (Thumb) Matsum \& Nakai, Cucumeropsis mannii and Lagenaria siceraria (Molina) Standl.Cv. Aklamkpa) in sociolinguistic areas in Benin. Biotechnologie, Agronomie, Societe et Environment 12: 393-403

Anonymous (2011). International Rules for Seed Testing. ISTA, Zurich, Switzerland

Huma Khan, Bedi Seema and Singh Rajinder 2016. Seed quality in pumpkin (Cucurbita pepo 1) cv. punjab samrat as affected by fruit harvesting and storage duration. Agricultural Research Journal 53(2): 220-23. 
Karklelienen R, Viskelis P and Rubinskeiene M. (2008). Growing, yielding and quality of different ecologically grown pumpkin cultivars. Scientific works of Lithuanium Institute of Horticulture. Lithuanium University of Agriculture. Sodininkyste 27:401-10

Nerson H and Paris HS(1988). Effect of fruit age, fermentation and storage on germination of cucurbit seeds. Sci Hort35: 15-26

Nerson, H. (2007). Seed production and germinability of cucurbit crops. Seed Science Biotechnology, Global Science Books, pp. 1-10

Oztokat C, Demir I and Kazim M 2004. Changes in germination and potential longevity of watermelon (Citrullus lanatus) seeds during development. $J$ Crop Hort Sci 32:139-45.

Raun S, Xue Q and Tylkowska K(2002). The influence of priming on germination of rice (Oryza sativa L.) seeds and seedling emergence and performance in flooded soil. Seed Sci Technol30:61-67

Singh, A.R. and Lachanna A 1995. Effects of date of harvesting, drying and storage on seed quality of sorghum parental lines. Seed Res13: 180-85

Tarus WJ, Ochuodho, J O and Rop, NK 2017. Influence of harvesting stage on seed quality aspects of pumpkin. Journal of Experimental Agricultural International 18(2): 1-9.

Welbaum, G. E. (1999). Cucurbit seed development and production. Hort Technol3:341-48

Wilson OO and Mcdonald MB(1986). The lipid peroxidation model of seed ageing. Seed Sci Technol14:269-300

Yao KB, Konan AJ, Coffi KK and Baudin JP (2012). Effect of fruit age, prestorage duration and seed fermentation durations on seed germination and seedling vigour in Lagenaria siceraria. J ApplBio Sci49: 3339-51.

\section{How to cite this article:}

Rajinder Singh, Parvinder Singh and Tarsem Singh Dhillon. 2020. Physiological Maturity and Longevity of Pumpkin Seeds in Relation to Fruit Age and Duration of in situ Storage. Int.J.Curr.Microbiol.App.Sci. 9(08): 2633-2641. doi: https://doi.org/10.20546/ijcmas.2020.908.301 